\title{
Chemical Evolution of Heavy Elements in the Early Galaxy: Implications for Stellar Sources
}

\author{
Yong-Zhong Qian ${ }^{\mathrm{A}}$ \\ A School of Physics and Astronomy, University of Minnesota, Minneapolis, MN 55455, USA. \\ Email: qian@physics.umn.edu
}

Received 2007 October 11, accepted 2008 January 14

\begin{abstract}
An overview of the sources for heavy elements in the early Galaxy is given. It is shown that observations of abundances in metal-poor stars can be used along with a basic understanding of stellar models to guide the search for the source of the heavy $r$-process nuclei ( $r$-nuclei). Observations show that this source produces very little of the elements from $\mathrm{C}$ through $\mathrm{Zn}$, including $\mathrm{Fe}$. This strongly suggests that $\mathrm{O}-\mathrm{Ne}-\mathrm{Mg}$ core-collapse supernovae ( $\mathrm{SNe}$ ) from progenitors of $\sim 8-11 \mathrm{M}_{\odot}$ are the source for the heavy $r$-nuclei. It is shown that a two-component model based on the abundances of Fe (from Fe core-collapse SNe) and Eu (from $\mathrm{O}-\mathrm{Ne}-\mathrm{Mg}$ core-collapse $\mathrm{SNe}$ ) gives very good quantitative predictions for the abundances of all the other elements in metal-poor stars.
\end{abstract}

Keywords: Galaxy: evolution — nuclear reactions, nucleosynthesis, abundances — stars: Population II — supernovae: general

\section{Introduction}

There are two approaches to studying chemical evolution. The straightforward approach relies on the knowledge of all nucleosynthetic sources, their elemental yields, their occurrences in the interstellar medium (ISM) and their exchange of material with the ISM. Based on this knowledge, both the average and the scatter for the abundance of any element in the ISM can be calculated as functions of time. Unfortunately, our knowledge of nucleosynthetic sources is rather incomplete. So this forward approach cannot be applied to all elements. On the other hand, the elemental abundances in stars generally reflect the composition of the ISM from which they formed. Given a large collection of data on stellar abundances, it is possible to infer the characteristics of some nucleosynthetic sources from these data with the help of some basic understanding of stellar models. This reverse approach works best at early times when only a small number of potential sources could have contributed to the ISM. This paper presents a combination of forward and reverse approaches to studying chemical evolution of heavy elements in the early Galaxy. The goal is to illustrate what can be learned about stellar sources from such studies.

Here 'early' means the first Gyr or so of Galactic history. During this epoch, only short-lived massive stars could have had time to evolve and contribute their nucleosynthetic products to the ISM. As $\sim 1 / 3$ of the solar Fe inventory was contributed by core-collapse supernovae (SNe, mostly Type II) associated with massive stars over a period of $\sim 10 \mathrm{Gyr}$, 'early times' also correspond to $<1 / 30$ of the solar $\mathrm{Fe}$ abundance in the ISM. In the notation $[\mathrm{Fe} / \mathrm{H}] \equiv \log (\mathrm{Fe} / \mathrm{H})-\log (\mathrm{Fe} / \mathrm{H})_{\odot}$, such times correspond to metal-poor ISM with $[\mathrm{Fe} / \mathrm{H}]<-1.5$. Three groups of heavy elements will be discussed: (1) the elements from $\mathrm{C}$ through $\mathrm{Zn}$ with mass numbers $A<70$, (2) the elements from $\mathrm{Sr}$ through $\mathrm{Ag}$ with $A \sim 88-110$ and (3) the elements with $A \sim 130$ through the actinides attributed to the rapid neutron capture process (the $r$ process). The solar abundances of the elements with $A \sim 88-209$ received important contributions from the slow neutron capture process (the $s$-process). However, as the major $s$-processing occurs in long-lived (with lifetimes of $>1 \mathrm{Gyr}$ ) stars of a few $\mathrm{M}_{\odot}$, the $s$-process contributions to the ISM at $[\mathrm{Fe} / \mathrm{H}]<-1.5$ can be ignored to good approximation. Likewise, contributions to the Fe group elements from Type Ia SNe associated with white dwarfs evolved from long-lived low-mass stars in binaries can also be ignored at $[\mathrm{Fe} / \mathrm{H}]<-1.5$.

\section{Elements from $\mathrm{C}$ through $\mathrm{Zn}$}

The relevant sources for the elements from $\mathrm{C}$ through $\mathrm{Zn}$ are massive stars of $>11 \mathrm{M}_{\odot}$, which give rise to $\mathrm{Fe}$ core-collapse $\mathrm{SNe}$ at the end of their evolution. The elements from $\mathrm{C}$ through $\mathrm{Al}$ are mainly produced by hydrostatic burning during the pre-SN evolution and the elements from $\mathrm{Si}$ through $\mathrm{Zn}$ are mainly produced by explosive burning associated with the propagation of the SN shock through the shells above the Fe core. While the production of all these elements has some important dependence on the initial metallicity of the SN progenitor through, for example mass loss, pre-SN density structure and details of the explosion, theoretical yields of individual elements are broadly similar over a wide range of 
metallicities and have been calculated by several groups (e.g. Woosley \& Weaver 1995; Chieffi \& Limongi 2004; Tominaga, Umeda \& Nomoto 2007). There is an approximate overall agreement between the theoretical yields averaged over the mass distribution of SN progenitors and the data (e.g. Cayrel et al. 2004) on the abundances of C through $\mathrm{Zn}$ in metal-poor stars with $-4<[\mathrm{Fe} / \mathrm{H}]<-3$ (e.g. Fröhlich et al. 2006a; Tominaga et al. 2007). However, there are also important deficiencies of the SN models. For example, the calculated abundance ratios of $\mathrm{N}, \mathrm{K}, \mathrm{Sc}, \mathrm{Ti}, \mathrm{Mn}$ and Co relative to $\mathrm{Fe}$ are too low compared with observations (e.g. Tominaga et al. 2007). Nitrogen is produced by the $\mathrm{CN}$ cycle when the $\mathrm{C}$ from He burning is mixed into the $\mathrm{H}$ burning shell. This production can be greatly enhanced by rotationally induced mixing when rotation is explicitly included in evolutionary models of metal-poor massive stars (e.g. Meynet, Ekström \& Maeder 2006). On the other hand, the underproduction of K, Sc, Ti, $\mathrm{Mn}$ and Co can be remedied by modifying, for example the electron fraction $Y_{\mathrm{e}}$ of the material undergoing explosive nucleosynthesis.

The electron fraction $Y_{\mathrm{e}}$ specifies the neutron-to-proton ratio of the material and plays a crucial role in nucleosynthesis. The conversion between neutrons and protons can only proceed through the weak interaction involving neutrinos. The death of massive stars can be considered as a neutrino phenomenon. When the Fe core of such a star collapses into a protoneutron star, a great amount of gravitational binding energy is released in $v_{\mathrm{e}}, \bar{v}_{\mathrm{e}}, v_{\mu}, \bar{v}_{\mu}$, $\nu_{\tau}$ and $\bar{v}_{\tau}$ with average energies of $\left\langle E_{v}\right\rangle \sim 10-20 \mathrm{MeV}$. Typical luminosity of the initial neutrino emission is $L_{v} \sim 10^{52} \mathrm{erg} \mathrm{s}^{-1}$ per species. In the case of stable protoneutron stars, neutrino emission with $L_{v} \sim 10^{51} \mathrm{erg} \mathrm{s}^{-1}$ per species lasts for $\sim 20 \mathrm{~s}$. With such intense neutrino fluxes, the neutron-to-proton ratio of the material close to the protoneutron star is modified by the reactions

$$
\begin{aligned}
& \bar{v}_{\mathrm{e}}+p \rightarrow n+e^{+}, \\
& \nu_{\mathrm{e}}+n \rightarrow p+e^{-} .
\end{aligned}
$$

The $Y_{\mathrm{e}}$ relevant for explosive nucleosynthesis in this material then depends on the competition between the above two reactions (e.g. Qian et al. 1993; Fuller \& Meyer 1995; Qian \& Woosley 1996). The rates of these reactions are proportional to the neutrino flux $L_{v} /\left\langle E_{v}\right\rangle$ and the cross section, which in turn is proportional to $\left\langle\left(E_{v} \mp \Delta\right)^{2}\right\rangle \approx\left\langle E_{v}^{2}\right\rangle \mp 2 \Delta\left\langle E_{v}\right\rangle$ with the minus sign being for reaction (1), the plus sign for reaction (2) and $\Delta=1.293 \mathrm{MeV}$ being the neutron-proton mass difference. When the reaction rates are sufficiently high, $Y_{\mathrm{e}}$ is determined by their ratio as (Qian \& Woosley 1996)

$$
Y_{\mathrm{e}} \approx\left[1+\frac{L_{\bar{\nu}_{\mathrm{e}}}}{L_{v_{\mathrm{e}}}}\left(\frac{\epsilon_{\bar{\nu}_{\mathrm{e}}}-2 \Delta}{\epsilon_{v_{\mathrm{e}}}+2 \Delta}\right)\right]^{-1},
$$

where $\epsilon_{v} \equiv\left\langle E_{v}^{2}\right\rangle /\left\langle E_{v}\right\rangle$.

As an example, the pairs of $\epsilon_{\bar{\nu}_{\mathrm{e}}}$ and $\epsilon_{\nu_{\mathrm{e}}}$ for $\bar{\nu}_{\mathrm{e}}$ and $v_{\mathrm{e}}$ emitted by the protoneutron star in the $\mathrm{SN}$ model of

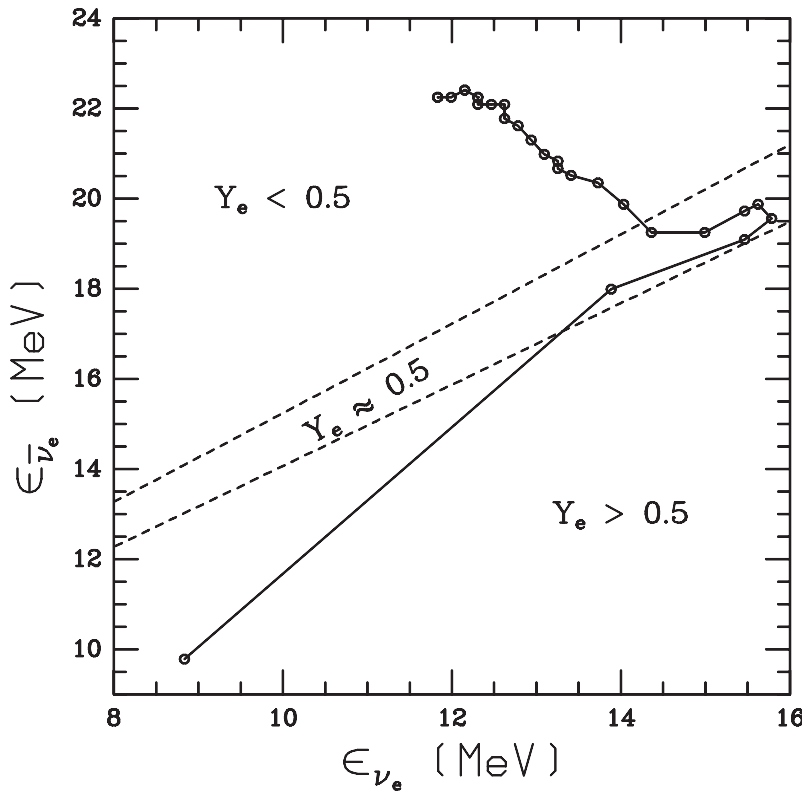

Figure 1 Example evolution of $\epsilon_{\bar{v}_{\mathrm{e}}}$ and $\epsilon_{v_{\mathrm{e}}}$ for $\bar{\nu}_{\mathrm{e}}$ and $\nu_{\mathrm{e}}$ emitted by a protoneutron star (solid curve). Time increases along the solid curve starting from the lower left end. The ratio $L_{\bar{v}_{\mathrm{e}}} / L_{v_{\mathrm{e}}}$ stays close to 1 throughout the evolution. Values of $\epsilon_{\bar{\nu}_{\mathrm{e}}}$ and $\epsilon_{\nu_{\mathrm{e}}}$ lying above the upper dashed line would give $Y_{\mathrm{e}}<0.5$ for $L_{\overline{\mathrm{v}}_{\mathrm{e}}} / L_{v_{\mathrm{e}}}=1$, those below the lower dashed line would give $Y_{\mathrm{e}}>0.5$ for $L_{\bar{v}_{\mathrm{e}}} / L_{v_{\mathrm{e}}}=1.1$ and those between the two dashed lines would give $Y_{\mathrm{e}} \approx 0.5$, with the exact $Y_{\mathrm{e}}$ being sensitive to the value of $L_{\bar{v}_{\mathrm{e}}} / L_{v_{\mathrm{e}}}$. See Qian \& Woosley (1996) for details.

Woosley et al. (1994) during the first $\sim 20$ s of its life are shown as the solid curve in Figure 1. The lower left end of the curve corresponds to the onset of the Fe core collapse at time $t \approx 0$. Time increases along the curve in intervals of $\approx 1 / 3 \mathrm{~s}$ for $t \approx 0-4 \mathrm{~s}$ and $\approx 1 \mathrm{~s}$ for $t>4 \mathrm{~s}$. The ratio $L_{\bar{v}_{\mathrm{e}}} / L_{v_{\mathrm{e}}}$ stays close to 1 throughout the evolution. Values of $\epsilon_{\bar{v}_{\mathrm{e}}}$ and $\epsilon_{v_{\mathrm{e}}}$ lying above the upper dashed line in Figure 1 would give $Y_{\mathrm{e}}<0.5$ for $L_{\bar{v}_{\mathrm{e}}} / L_{\nu_{\mathrm{e}}}=1$ according to equation (3), those below the lower dashed line would give $Y_{\mathrm{e}}>0.5$ for $L_{\bar{v}_{\mathrm{e}}} / L_{v_{\mathrm{e}}}=1.1$ and those between the two dashed lines would give $Y_{\mathrm{e}} \approx 0.5$, with the exact $Y_{\mathrm{e}}$ being sensitive to the value of $L_{\bar{v}_{\mathrm{e}}} / L_{v_{\mathrm{e}}}$.

During the first $\sim 1 \mathrm{~s}$ after the onset of Fe core collapse, the neutrino emission characteristics $L_{\bar{v}_{\mathrm{e}}} \approx L_{v_{\mathrm{e}}}$ and $\epsilon_{\bar{v}_{\mathrm{e}}}<\epsilon_{\mathrm{v}_{\mathrm{e}}}+4 \Delta$ (i.e. below the upper dashed line in Figure 1) give rise to $Y_{\mathrm{e}}>0.5$ in the material immediately above the protoneutron star. This results in greatlyenhanced yields of ${ }^{45} \mathrm{Sc}$, ${ }^{49} \mathrm{Ti}$ and ${ }^{64} \mathrm{Zn}$, due to the production of their more proton-rich progenitor nuclei ${ }^{45} \mathrm{Cr},{ }^{45} \mathrm{~V},{ }^{49} \mathrm{Mn}$ and ${ }^{64} \mathrm{Ge}$ (Pruet et al. 2005; Fröhlich et al. 2006a). In particular, it was shown that the inclusion of neutrino effects on $Y_{\mathrm{e}}$ dramatically increases the calculated abundance ratios $\mathrm{Sc} / \mathrm{Fe}$ and $\mathrm{Zn} / \mathrm{Fe}$ to the level in accordance with observations of metal-poor stars with $-4.1<[\mathrm{Fe} / \mathrm{H}]<-0.8$ (Fröhlich et al. 2006a). Therefore, it appears that $\mathrm{Fe}$ core-collapse $\mathrm{SNe}$ from progenitors of $>11 \mathrm{M}_{\odot}$ are reasonably established as the major source for the elements from $\mathrm{C}$ through $\mathrm{Zn}$ in the early Galaxy. 


\section{Production of Heavy Elements in the Neutrino-Driven Wind}

Reactions (1) and (2) not only are important for determining the $Y_{\mathrm{e}}$ of the material close to the protoneutron star, but also heat this material, enabling it to expand away from the protoneutron star as a neutrino-driven wind (e.g. Qian \& Woosley 1996). In fact, as long as a stable protoneutron star is formed by some core collapse, its neutrino emission drives such a wind for $\sim 20 \mathrm{~s}$. As the wind expands away from the protoneutron star, neutrinos can continue to affect nucleosynthesis. For example, the nuclear flow in proton-rich conditions encounters bottlenecks at nuclei with extremely slow proton-capture and $\beta^{+}$-decay rates. In the presence of an intense $\bar{v}_{\mathrm{e}}$ flux, the neutrons produced by reaction (1) can be captured by such nuclei to break through the bottleneck, giving rise to the so-called $v p$ process (Fröhlich et al. 2006b), which can produce many nuclei beyond ${ }^{64} \mathrm{Zn}$. It is important to find out whether the observed correlation between the abundances of $\mathrm{Ge}$ and $\mathrm{Fe}$ (Cowan et al. 2005) can be accounted for after the contribution to Ge from the $v p$-process is taken into account. General studies of nucleosynthesis in the proton-rich neutrino-driven wind were carried out in detail by Fröhlich et al. (2006b), Pruet et al. (2006) and Wanajo (2006).

As shown in Figure 1, the difference between $\epsilon_{\bar{v}_{\mathrm{e}}}$ and $\epsilon_{v_{\mathrm{e}}}$ increases with time and the neutrino-driven wind eventually becomes neutron-rich (i.e. $Y_{\mathrm{e}}<0.5$ ). What can be produced in a neutron-rich wind? In general, nucleosynthesis during expansion of material from an initial state of high temperature depends on $Y_{\mathrm{e}}$, the entropy $S$ and the dynamic expansion timescale $\tau_{\text {dyn }}$ of this material. In the neutrino-driven wind, these three parameters are determined by the mass, radius and neutrino emission characteristics of the protoneutron star (e.g. Qian \& Woosley 1996). As the neutrino emission characteristics evolve, the conditions in the wind change accordingly. For illustration, we consider two sets of conditions corresponding to winds taking off at two different times from a protoneutron star of $1.4 \mathrm{M}_{\odot}$ with a radius of $10 \mathrm{~km}$. At an earlier time, the wind has $Y_{\mathrm{e}} \approx 0.47, S \approx 70$ (in units of Boltzmann constant per nucleon) and $\tau_{\text {dyn }} \approx 0.024 \mathrm{~s}$. As the neutron-rich material in this wind expands, the free nucleons first combine into $\alpha$-particles. This consumes essentially all the protons. Then an $\alpha$-process occurs to burn $\alpha$-particles and the remaining neutrons into heavier nuclei (Woosley \& Hoffman 1992). By the time all charged-particle reactions (CPRs) cease at sufficiently low temperature due to the Coulomb barrier, the dominant products are $\mathrm{Sr}, \mathrm{Y}$ and $\mathrm{Zr}$ with $A \sim 90$ and no neutrons are left to further process these nuclei by neutron capture (Hoffman, Woosley \& Qian 1997). For a late wind with $Y_{\mathrm{e}} \approx 0.37, S \approx 90$ and $\tau_{\mathrm{dyn}} \approx 0.066 \mathrm{~s}$, the dominant products are $\mathrm{Zr}, \mathrm{Mo}, \mathrm{Ru}, \mathrm{Rh}, \mathrm{Pd}$ and $\mathrm{Ag}$ with $A \sim 96-110$ (Hoffman et al. 1997). These nuclei are also produced by CPRs during the $\alpha$-process and no neutrons are left at the end. As another example, we consider a late wind from a heavier protoneutron star of $2 \mathrm{M}_{\odot}$ but with the same radius of $10 \mathrm{~km}$. The conditions of $Y_{\mathrm{e}} \approx 0.35, S \approx 140$ and $\tau_{\text {dyn }} \approx 0.11 \mathrm{~s}$ in this wind result in major production of $\mathrm{Sn}, \mathrm{Sb}$ and Te, with $A \sim 124$ in addition to the nuclei with $A \sim 96-110$. Once again, all these nuclei are produced by CPRs during the $\alpha$-process and few neutrons are left at the end (Hoffman et al. 1997).

The above discussion shows that for the typical conditions in a neutron-rich wind, elements from $\mathrm{Sr}$ through $\mathrm{Ag}$ with $A \sim 88-110$ and in some cases nuclei with $A \sim 124$, are produced by CPRs during the $\alpha$-process. As no or very few neutrons are left at the end of the $\alpha$-process, these nuclei are the main products in such winds. In contrast, if the abundance ratio of neutrons to heavy nuclei greatly exceeds $\sim 10$ when CPRs cease to occur at a temperature of several $10^{9} \mathrm{~K}$ due to the Coulomb barrier, then the $\alpha$-process smoothly merges with the $r$-process as the heavy nuclei produced by the former rapidly capture neutrons at lower temperatures. This is the neutrino-driven wind model for the $r$-process (e.g. Woosley \& Baron 1992; Meyer et al. 1992; Takahashi, Witti \& Janka 1994; Woosley et al. 1994; Wanajo \& Ishimaru 2006). In this model, the final abundance pattern produced by the $r$ process depends on how many neutrons are left for each seed nucleus at the end of the $\alpha$-process. For example, a neutron-to-seed ratio of $n / s \sim 40$ would dominantly produce an abundance peak at $A \sim 130$ while another value of $n / s \sim 90$ would dominantly produce nuclei of $A>130$ with an abundance peak at $A \sim 195$.

In general, a specific value of $n / s$ can be achieved by various combinations of $Y_{\mathrm{e}}, S$ and $\tau_{\mathrm{dyn}}$. A lower $Y_{\mathrm{e}}$ corresponds to a higher initial neutron abundance and usually also means that more neutrons are left at the end of the $\alpha$-process. A value of $S>10$ indicates that the energy density of the material is dominated by radiation (and electron-positron pairs for sufficiently high temperature). The production of seed nuclei is severely suppressed for $S>100$. This is because in producing the seed nuclei the nuclear flow must rely on the three-body reaction $\alpha+\alpha+n \rightarrow{ }^{9} \mathrm{Be}+\gamma$ to bridge the gap at $A=5$ and 8 . It only requires a photon of $1.573 \mathrm{MeV}$ to dissociate a ${ }^{9} \mathrm{Be}$ nucleus back into two $\alpha$-particles and a neutron. For a high $S$, there are a significant number of such photons in the high-energy tail of the Bose-Einstein distribution over the temperature range of $\sim 3-6 \times 10^{9} \mathrm{~K}$ for the $\alpha$-process. The dynamic timescale $\tau_{\text {dyn }}$ controls how fast the temperature drops, thereby specifying the duration of the $\alpha$-process. Clearly, the shorter $\tau_{\text {dyn }}$ is, the fewer seed nuclei are produced. In summary, a lower $Y_{\mathrm{e}}$, or a higher $S$, or a shorter $\tau_{\text {dyn }}$ tends to give a larger $n / s$.

Combinations of $Y_{\mathrm{e}}$ and $S$ that would result in major production of an $r$-process abundance peak at $A \sim 195$ for three different values of $\tau_{\text {dyn }}$ (Hoffman et al. 1997; see also Meyer \& Brown 1997; Freiburghaus et al. 1999) are shown in Figure 2. It can be seen that for $Y_{\mathrm{e}} \sim 0.4$ and $S \sim 100$ typical of the neutrino-driven wind, an extremely short dynamic timescale of $\tau_{\text {dyn }} \sim 0.004 \mathrm{~s}$ is required to produce an $r$-process abundance peak at $A \sim 195$. 


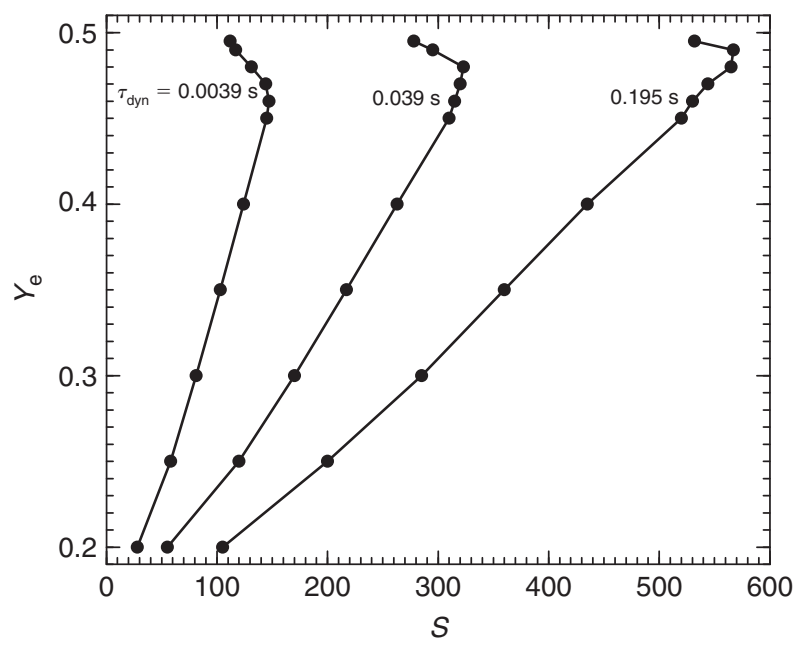

Figure 2 Combinations of $Y_{\mathrm{e}}$ and $S$ that would result in major production of an $r$-process abundance peak at $A \sim 195$ during expansion of material from an initial state of high temperature for three values of $\tau_{\mathrm{dyn}}$. See Hoffman et al. (1997) for details.

However, the actual dynamic timescale typical of the wind is $\tau_{\text {dyn }} \sim 0.01-0.1 \mathrm{~s}$. Studies by several groups (Qian \& Woosley 1996; Witti et al. 1994; Thompson, Burrows \& Meyer 2001) found that it is generally very difficult to obtain the conditions required for producing an $r$-process abundance peak at $A \sim 195$ in the neutrino-driven wind, although it is quite plausible that the conditions in the wind from at least some protoneutron stars (e.g. those with masses of $\sim 2 \mathrm{M}_{\odot}$ ) are sufficient for producing $r$-process nuclei up to $A \sim 130$.

\section{Source for $r$-Process Nuclei with $A>130$}

In search of the source for the heavy $r$-process nuclei (r-nuclei) with $A>130$, especially those in the peak at $A \sim 195$ of the solar $r$-process abundance pattern ( $r$ pattern; e.g. Arlandini et al. 1999), there were attempts to modify the conditions in the neutrino-driven wind by, for example, including the effects of a magnetic field above the protoneutron star (Thompson 2003) as well as proposals of alternative sites such as neutron star mergers (e.g. Freiburghaus, Rosswog \& Thielmann 1999) and the wind from the accretion disk around a black hole (Pruet et al. 2003; McLaughlin \& Surman 2005). Yet another approach is to seek guidance from observations. For example, the detection of the $r$-process element ( $r$-element) Ba with $A \sim 135$ in a number of stars with $[\mathrm{Fe} / \mathrm{H}]<-3$, especially the high $\mathrm{Ba}$ enrichments in several stars with $[\mathrm{Fe} / \mathrm{H}] \sim-3$, is crucial in evaluating neutron star mergers as the major source for the heavy $r$-nuclei. These events are much rarer (by at least a factor of $10^{3}$ ) than Fe core-collapse SNe. If neutron star mergers were the major source for the heavy $r$-nuclei, then enrichment in these nuclei would not occur until the ISM had already been substantially enriched in Fe by Fe core-collapse SNe (Qian 2000; Argast et al. 2004). This is in contradiction to the observations of stars with significant to high $\mathrm{Ba}$ abundances but very low Fe abundances. Therefore, it appears very unlikely that neutron
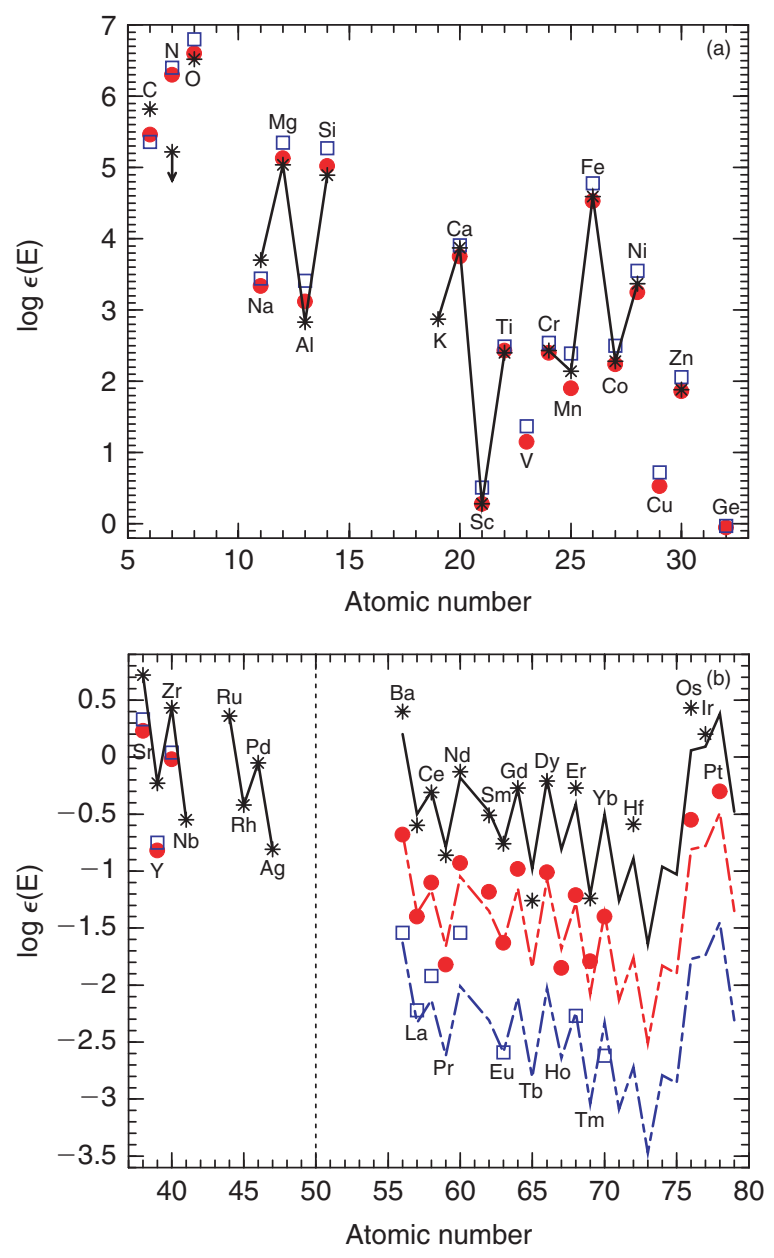

Figure 3 Data on the elements from C through Pt in CS 31082-001 (asterisks; Hill et al. 2002), HD 115444 (filled circles) and HD 122563 (squares; Westin et al. 2000) with $[\mathrm{Fe} / \mathrm{H}]=-2.9,-2.99$ and -2.74 , respectively. (a) The values of $\log \epsilon(\mathrm{E}) \equiv \log (\mathrm{E} / \mathrm{H})+12$ for the elements from $\mathrm{C}$ through $\mathrm{Ge}$. The data on CS 31082-001 are connected by solid line segments as a guide. The downward arrow at the asterisk for $\mathrm{N}$ indicates an upper limit. Note that the available abundances for the elements from $\mathrm{O}$ through $\mathrm{Ge}$ are almost indistinguishable for the three stars. (b) The $\log \epsilon$ values for the elements from Sr through Pt. The data for CS 31082-001 in the region to the left of the vertical dotted line are again connected by solid line segments as a guide. In the region to the right of the vertical dotted line, the data on the heavy $r$-elements are compared with the solid, dot-dashed and dashed curves, which are the solar $r$-pattern (Arlandini et al. 1999) translated to pass through the Eu data for CS 31082-001, HD 115444 and HD 122563, respectively. Note the general agreement between the data and the solid and dot-dashed curves. There is a range of $\sim 2$ dex in the abundances of the heavy $r$-elements for the three stars shown.

star mergers are the major source for the heavy $r$-nuclei. In contrast, if some events with a Galactic rate of occurrences similar to that of $\mathrm{Fe}$ core-collapse $\mathrm{SNe}$ are the source for such nuclei, then the expected relationship between $\mathrm{Ba}$ and $\mathrm{Fe}$ abundances is in good agreement with stellar observations (e.g. Argast et al. 2004).

Extensive observations of the abundances of a wide range of elements in metal-poor stars provide further guidance to the search of the source for the heavy $r$ nuclei. Figure $3 b$ shows the data on the elements from $\mathrm{Sr}$ 
through Pt for CS 31082-001 (asterisks; Hill et al. 2002), HD 115444 (filled circles) and HD 122563 (squares; Westin et al. 2000). The curves to the right of the vertical dotted line represent the solar $r$-pattern translated to pass through the Eu data. It can be seen that the data on the heavy $r$-elements from Ba through Pt in CS 31082-001 and HD 115444 closely follow the solar $r$-pattern. There are fewer data on the heavy $r$-elements in HD 122563 and this star will be discussed in more detail in Section 5. Note that the levels of enrichment in the heavy $r$-elements differ by a factor of $\sim 100$ for the three stars shown in Figure 3b. However, their abundances of the elements from $\mathrm{C}$ through $\mathrm{Ge}$ are essentially identical (e.g. $[\mathrm{Fe} / \mathrm{H}] \approx-3$ ) as shown in Figure $3 \mathrm{a}$. This appears to require that the major source for the heavy $r$-nuclei produce none of the elements from $\mathrm{C}$ through $\mathrm{Ge}$ including $\mathrm{Fe}$ (Qian \& Wasserburg 2002, 2003, 2007). This decoupling of the production of the heavy $r$-nuclei from that of the elements from $\mathrm{C}$ through $\mathrm{Ge}$ is further demonstrated by comparing CS 22892-052 $([\mathrm{Fe} / \mathrm{H}]=-3.1$, Sneden et al. 2003) with HD $221170([\mathrm{Fe} / \mathrm{H}]=-2.2$, Ivans et al. 2006) and CS 31082-001 $([\mathrm{Fe} / \mathrm{H}]=-2.9)$ with $\mathrm{BD}+17^{\circ} 3248$ $([\mathrm{Fe} / \mathrm{H}]=-2.1$; Cowan et al. 2002). As shown in Figure 4, the stars in either pair have nearly the same abundances of the heavy $r$-elements, which again closely follow the solar $r$-pattern. However, as shown by the data on these stars (Hill et al. 2002; Cowan et al. 2002; Sneden et al. 2003; Ivans et al. 2006) and reflected by their [Fe/H] values, the abundances of the elements between $\mathrm{O}$ and $\mathrm{Ge}$ differ by a factor of $\sim 8$ and 6 for the former and latter pair, respectively. Therefore, the decoupling between the heavy $r$-nuclei and the elements from $\mathrm{C}$ through $\mathrm{Ge}$ appears to be complete: the major source for the former group of nuclei produces very little of the latter while the major source for the latter produces very little of the former.

The elements from $\mathrm{C}$ through $\mathrm{Zn}$ are produced between the core and the $\mathrm{H}$ envelope by explosive burning during a core-collapse $\mathrm{SN}$ or by hydrostatic burning during the pre-SN evolution. Stars of $>11 \mathrm{M}_{\odot}$ develop Fe cores surrounded by extensive shells of $\mathrm{Si}, \mathrm{O}, \mathrm{C}$ and $\mathrm{He}$. Consequently, Fe core-collapse $\mathrm{SNe}$ from these stars are the major source for the elements from $\mathrm{C}$ through $\mathrm{Zn}$ in the early Galaxy. The decoupling between these elements and the heavy $r$-nuclei discussed above then strongly suggests that such $\mathrm{SNe}$ are not the source for the heavy $r$ nuclei. In contrast, stars of $\sim 8-11 \mathrm{M}_{\odot}$ develop degenerate $\mathrm{O}-\mathrm{Ne}-\mathrm{Mg}$ cores, at least some of which eventually collapse to produce $\mathrm{SNe}$ (e.g. Nomoto 1984, 1987; Ritossa, García-Berro \& Iben 1999). Models of O-Ne-Mg corecollapse SNe show that the total amount of material ejected from between the core and the $\mathrm{H}$ envelope is only 0.01-0.04 $\mathrm{M}_{\odot}$ (Mayle \& Wilson 1988; Kitaura, Janka \& Hillebrandt 2006), much smaller than the $\sim 1 \mathrm{M}_{\odot}$ for $\mathrm{Fe}$ core-collapse SNe. Thus, O-Ne-Mg core-collapse $\mathrm{SNe}$ contribute very little to the elements from $\mathrm{C}$ through $\mathrm{Zn}$. The decoupling between these elements and the heavy $r$-nuclei can then be explained by attributing the heavy
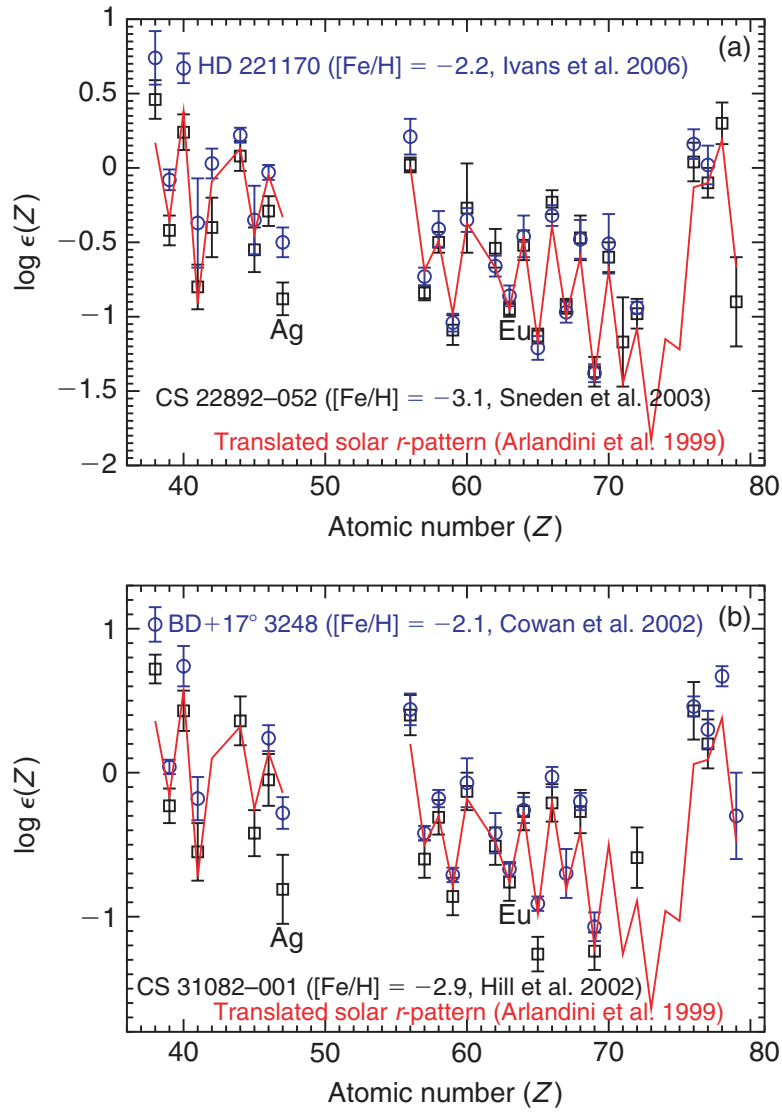

Figure 4 Comparison of abundances in two pairs of metal-poor stars. (a) CS 22892-052 with $[\mathrm{Fe} / \mathrm{H}]=-3.1$ (squares with error bars; Sneden et al. 2003) and HD 221170 with $[\mathrm{Fe} / \mathrm{H}]=-2.2$ (circles with error bars; Ivans et al. 2006). (b) CS 31082-001 with $[\mathrm{Fe} / \mathrm{H}]=-2.9$ (squares with error bars; Hill et al. 2002) and BD $+17^{\circ} 3248$ with $[\mathrm{Fe} / \mathrm{H}]=-2.1$ (circles with error bars; Cowan et al. 2002). The curves in (a) and (b) give the solar ' $r$-pattern' (Arlandini et al. 1999) translated to pass through the Eu data for CS 22892-052 and CS 31082-001, respectively. Note that each pair of stars have nearly identical abundances of the heavy $r$-elements closely following the solar $r$-pattern but the $(\mathrm{Fe} / \mathrm{H})$ values differ by a factor of 8 and 6 for the pair in (a) and (b), respectively. In addition, for an element $\mathrm{E}$ in the group from $\mathrm{Na}$ through $\mathrm{Zn}$, the abundance ratio $(\mathrm{E} / \mathrm{Fe})$ is the same for the four stars shown in (a) and (b) when observational errors are taken into account.

$r$-nuclei to such SNe as argued in Qian and Wasserburg (2002, 2003, 2007).

$\mathrm{O}-\mathrm{Ne}-\mathrm{Mg}$ core-collapse $\mathrm{SNe}$ were also proposed as the source for the heavy $r$-nuclei based on considerations of Galactic chemical evolution by other studies (e.g. Mathews, Bazan \& Cowan 1992; Ishimaru \& Wanajo 1999). This proposal is supported by the model for $r$-process nucleosynthesis presented in Ning, Qian \& Meyer (2007). Unlike previous models based on assumed extremely neutron-rich ejecta (e.g. Wheeler, Cowan \& Hillebrandt 1998; Wanajo et al. 2003), this new model relies on the $\mathrm{SN}$ shock that rapidly accelerates through the surface $\mathrm{C}-\mathrm{O}$ layers of the $\mathrm{O}-\mathrm{Ne}-\mathrm{Mg}$ core due to the steep density fall-off in these layers. This gives rise to fast expansion of the shocked ejecta on dynamic timescales of $\tau_{\mathrm{dyn}} \sim 10^{-4}$ s. Together with an entropy of $S \sim 100$ and 
Table 1. Stellar sources for heavy elements

\begin{tabular}{lll}
\hline & $>11 \mathrm{M}_{\odot}{ }^{\mathrm{a}}$ & $\sim 8-11 \mathrm{M}_{\odot}{ }^{\mathrm{b}}$ \\
\hline $\mathrm{C}$ to $\mathrm{Zn}(A<70)$ & Yes & No \\
$\mathrm{Sr}$ to $\mathrm{Ag}(A \sim 88-110)$ & Yes & Yes \\
$r$-nuclei $(A \sim 130)$ & Maybe & $?$ \\
$r$-nuclei $(A>130)$ & No & Yes \\
\hline
\end{tabular}

${ }^{\text {a }}$ Progenitors of Fe core-collapse SNe.

${ }^{\mathrm{b}}$ Progenitors of $\mathrm{O}-\mathrm{Ne}-\mathrm{Mg}$ core-collapse $\mathrm{SNe}$.

an initial electron fraction of $Y_{\mathrm{e}} \sim 0.495$ (e.g. for a composition of ${ }^{13} \mathrm{C}:{ }^{12} \mathrm{C}:{ }^{16} \mathrm{O} \sim 1: 3: 3$ by mass), this fast expansion enables an $r$-process to occur in the shocked ejecta, producing nuclei with $A>130$ through the actinides. To further test this model requires two lines of important studies: (1) calculating the evolution of $\sim 8-11 \mathrm{M}_{\odot}$ stars to determine the pre-SN conditions of $\mathrm{O}-\mathrm{Ne}-\mathrm{Mg}$ cores, especially the neutron excess and density structure of the surface layers; and (2) simulating the collapse of such cores and the subsequent shock propagation to determine the conditions of the shocked surface layers. As these layers contain very little mass, simulations with extremely fine mass resolutions are required to demonstrate the fast expansion of shocked ejecta that is the key to the production of the heavy $r$-nuclei in the above model.

\section{Conclusions}

As summarized in Table 1, considerations of chemical evolution of heavy elements in the early Galaxy based on stellar observations and current understanding of stellar models show that (1) Fe core-collapse $\mathrm{SNe}$ from progenitors of $>11 \mathrm{M}_{\odot}$ are the major source for the elements from $\mathrm{C}$ through $\mathrm{Zn}$ in the early Galaxy; (2) O-Ne-Mg corecollapse $\mathrm{SNe}$ from progenitors of $\sim 8-11 \mathrm{M}_{\odot}$ are the major source for the heavy $r$-nuclei with $A>130$; and (3) as both $\mathrm{Fe}$ and $\mathrm{O}-\mathrm{Ne}-\mathrm{Mg}$ core-collapse $\mathrm{SNe}$ can produce protoneutron stars that emit neutrinos to drive winds, both kinds of SNe can produce the elements from $\mathrm{Sr}$ through $\mathrm{Ag}$ by CPRs during the $\alpha$-process. The neutrino-driven winds in some Fe core-collapse $\mathrm{SNe}$ (e.g. those producing heavier protoneutron stars) may even produce $r$-nuclei with $A \sim 130$ (corresponding to the elements Te, I and Xe), but stellar observations cannot help us to identify the sources for these nuclei as they are inaccessible to spectroscopic studies.

Based on the above attribution, one may try to identify two templates for the overall production of heavy elements by $\mathrm{Fe}$ and $\mathrm{O}-\mathrm{Ne}-\mathrm{Mg}$ core-collapse $\mathrm{SNe}$, respectively. The squares with error bars in Figure 5a show the data on the elements from Sr through Ir in HD 122563 (Honda et al. 2006). These data are linked by line segments to guide the eye. The solar ' $r$-pattern' translated to pass through the Eu data is shown as the curves labelled so in Figure 5a. It can be seen that the data on the heavy $r$-elements $\mathrm{Ce}$, $\mathrm{Pr}, \mathrm{Nd}$ and $\mathrm{Sm}$ lie significantly above the translated solar ' $r$-pattern' while those on the elements from Sr through
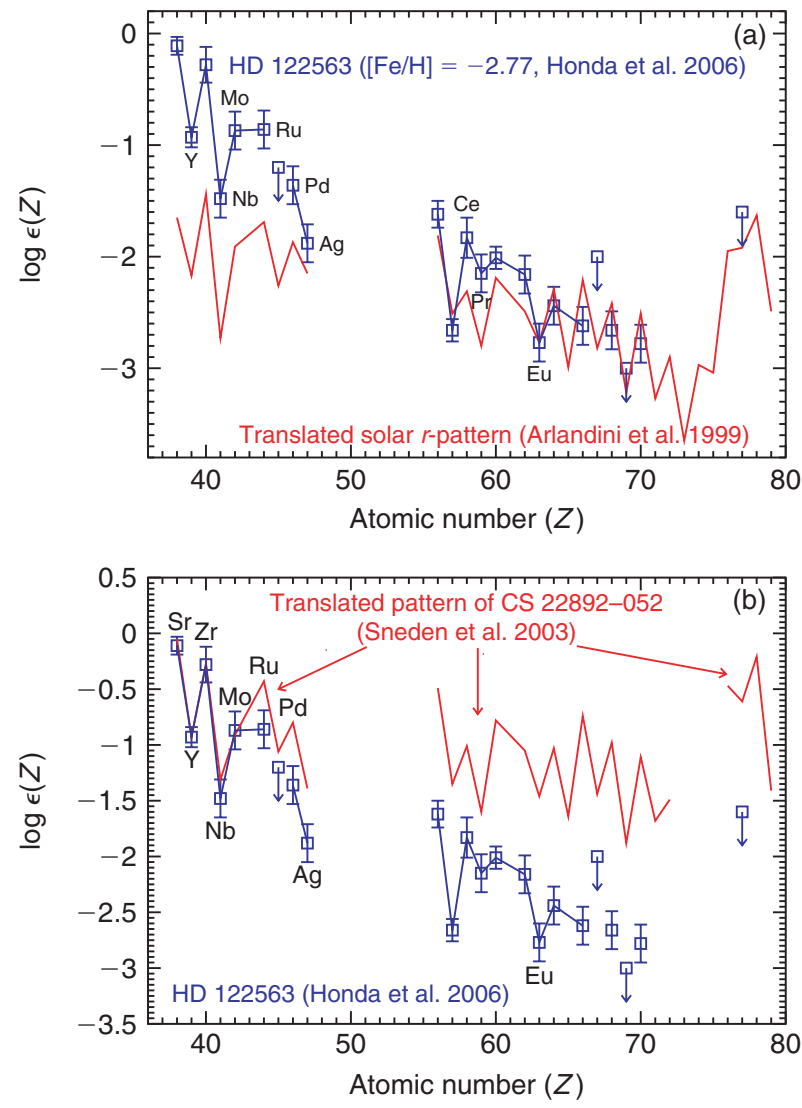

Figure 5 (a) Data on HD 122563 (squares with error bars linked by line segments; Honda et al. 2006) compared with the solar ' $r$-pattern' translated to pass through the Eu data (curves labelled as such). Squares with downward arrows indicate upper limits. The abundance pattern of the heavy $r$-elements $[\mathrm{Ba}(Z=56)$ and above] in HD 122563 shown in (a) exhibits substantial differences from the solar ' $r$-pattern', especially for $\mathrm{Ce}$ and Pr. In addition, HD 122563 has much larger proportions of the elements from $\mathrm{Sr}$ through Ag relative to the heavy $r$-nuclei as compared to the solar ' $r$-pattern'. (b) Comparison of the data on HD 122563 (squares with error bars linked by line segments; Honda et al. 2006) with those on CS 22892-052 (curves labelled as such; Sneden et al. 2003) normalized to the same $\log \epsilon(\mathrm{Y})$ as for HD 122563.

Ag lie far above it. This suggests that the source responsible for the abundances in HD 122563 is not a major contributor to the heavy $r$-elements but mainly produces the elements from $\mathrm{Sr}$ through Ag. Consequently, the overall abundance pattern in this star including the elements from $\mathrm{C}$ through $\mathrm{Zn}$ may be taken as representative of the yields of Fe core-collapse SNe. For convenience, this pattern will be referred to as the $L$-pattern (dominated by lighter nuclei). As Fe core-collapse $\mathrm{SNe}$ are the major source for Fe in the early Galaxy, the Fe abundance in a metal-poor star can be used along with the $L$-pattern to identify the absolute contributions from such $\mathrm{SNe}$ to all the elements in this star.

As shown in Figures 3 and 4, a number of metalpoor stars exhibit a highly regular abundance pattern of the heavy $r$-elements that is essentially identical to the solar $r$-pattern. The data on one such star, CS 22892 052, which are shifted to pass through the $\mathrm{Y}$ data for 

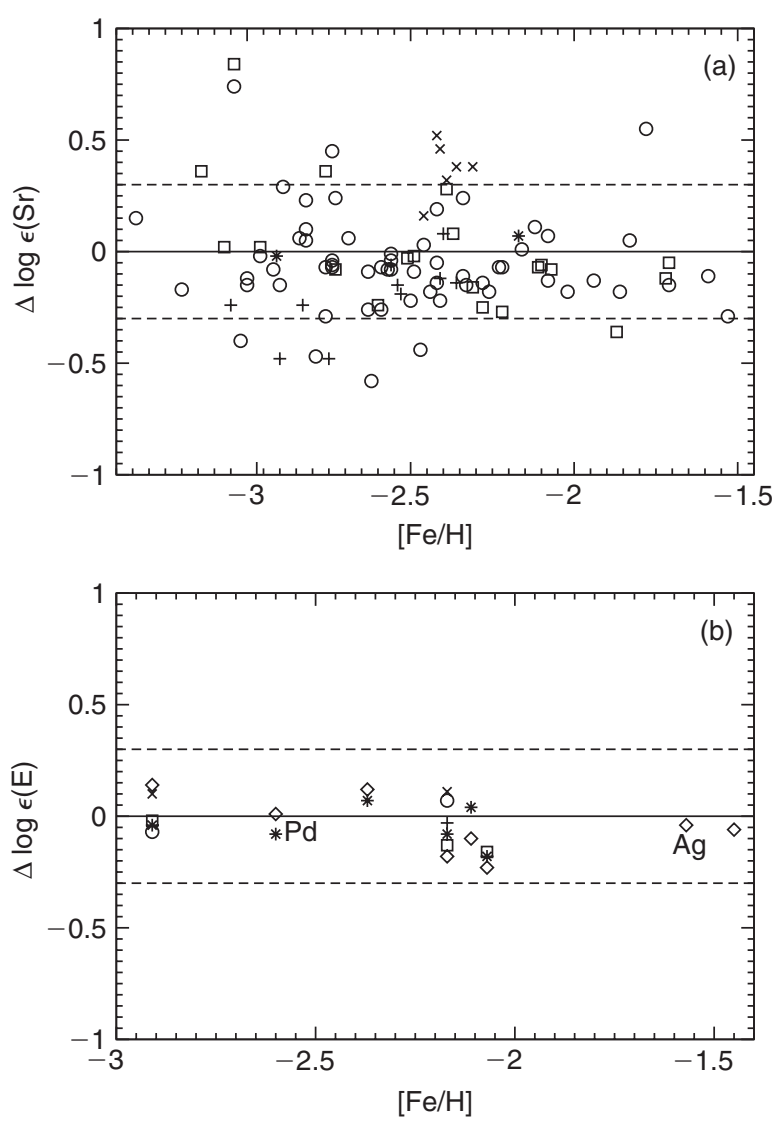

Figure 6 Comparison of the two-component model based on equation (4) with the data for a large sample of stars. (a) The difference between the calculated abundance of $\mathrm{Sr}$ and the observed value is shown in terms of $\Delta \log \epsilon(\mathrm{Sr}) \equiv \log \epsilon_{\mathrm{cal}}(\mathrm{Sr})-\log \epsilon_{\mathrm{obs}}(\mathrm{Sr})$ as a function of $[\mathrm{Fe} / \mathrm{H}]$. The calculation uses only the observed $\mathrm{Fe}$ and $\mathrm{Eu}$ abundances. The symbols represent the data sets as follows: squares for Johnson \& Bolte (2002), pluses for Aoki et al. (2005), circles for Barklem et al. (2005), crosses for Otsuki et al. (2006) and asterisks for Hill et al. (2002) and Ivans et al. (2006). (b) Comparison between the model and data on $\mathrm{Nb}$ (squares), Mo (pluses), $\mathrm{Ru}$ (circles), $\mathrm{Rh}$ (crosses), Pd (asterisks) and Ag (diamonds). The limited data available are taken from Crawford et al. (1998); Hill et al. (2002); Cowan et al. (2002); Johnson \& Bolte (2002); and Ivans et al. (2006). It can be seen from these examples that the elemental abundances in a metal-poor star are rather well estimated from the model using only the $\mathrm{Fe}$ and $\mathrm{Eu}$ abundances to identify the contributions from two different sources to the star.

HD 122563 for comparison with the latter star, are shown as the curves labelled so in Figure 5b. The Fe abundance in CS 22892-052 indicates that Fe core-collapse SNe contributed very little to its abundances of $\mathrm{Sr}$ and heavier elements. Figure $5 \mathrm{~b}$ shows that if the source responsible for the abundances of these elements in CS 22892-052 produces the elements from $\mathrm{Sr}$ through $\mathrm{Ag}$ at a similar level to an Fe core-collapse $\mathrm{SN}$, then this source must be the major source for the heavy $r$-elements. Consequently, the abundance pattern of $\mathrm{Sr}$ and heavier elements in CS 22892-052 can be taken as representative of the yields of $\mathrm{O}-\mathrm{Ne}-\mathrm{Mg}$ core-collapse SNe. For convenience, this pattern will be referred to as the $H$-pattern (dominated by heavier nuclei). As $\mathrm{O}-\mathrm{Ne}-\mathrm{Mg}$ core-collapse $\mathrm{SNe}$ are the major source for the heavy $r$-element $\mathrm{Eu}$, the $\mathrm{Eu}$ abundance in a metal-poor star can be used along with the $H$-pattern to identify the absolute contributions from such SNe to all the elements in this star.

Based on the above discussion, the abundance of element $\mathrm{E}$ in a metal-poor star can be determined as (Qian \& Wasserburg 2007)

$$
\left(\frac{\mathrm{E}}{\mathrm{H}}\right)=\left(\frac{\mathrm{E}}{\mathrm{Fe}}\right)_{L}\left(\frac{\mathrm{Fe}}{\mathrm{H}}\right)+\left(\frac{\mathrm{E}}{\mathrm{Eu}}\right)_{H}\left(\frac{\mathrm{Eu}}{\mathrm{H}}\right),
$$

where $(\mathrm{E} / \mathrm{Fe})_{L}$ and $(\mathrm{E} / \mathrm{Eu})_{H}$ are the yield ratios representing the $L$-pattern and the $H$-pattern taken from the data on HD 122563 (Honda et al. 2006) and CS 22892052 (Sneden et al. 2003), respectively. As an example, the abundances of $\mathrm{Sr}$ for a large number of metal-poor stars are calculated from equation (4) using the observed $\mathrm{Fe}$ and $\mathrm{Eu}$ abundances and compared with the data in terms of $\Delta \log \epsilon(\mathrm{Sr}) \equiv \log \epsilon(\mathrm{Sr})_{\mathrm{cal}}-\log \epsilon(\mathrm{Sr})_{\text {obs }}$ in Figure 6a. It can be seen that the calculated $\mathrm{Sr}$ abundances are within 0.3 dex of the data for the majority of the stars. With the limited data available for the elements $\mathrm{Nb}, \mathrm{Mo}, \mathrm{Ru}, \mathrm{Rh}$, $\mathrm{Pd}$ and $\mathrm{Ag}$, even better agreement between the calculated and observed abundances is obtained for these elements as shown in Figure 6b. In conclusion, the two-component model based on equation (4) appears to provide a very good description of the abundances in metal-poor stars. It remains to be seen whether self-consistent ab initio models of stellar evolution and nucleosynthesis can show that the $L$-pattern and the $H$-pattern are indeed characteristic of the yields of $\mathrm{Fe}$ and $\mathrm{O}-\mathrm{Ne}-\mathrm{Mg}$ core-collapse $\mathrm{SNe}$, respectively.

\section{Acknowledgments}

This work was supported in part by the US Department of Energy under grant DE-FG02-87ER40328.

\section{References}

Aoki, W. et al., 2005, ApJ, 632, 611

Argast, D., Samland, M., Thielemann, F.-K. \& Qian, Y.-Z., 2004, A\&A, 416, 997

Arlandini, C., Käppeler, F., Wisshak, K., Gallino, R., Lugaro, M., Busso, M. \& Straniero, O., 1999, ApJ, 525, 886

Barklem, P. S. et al., 2005, A\&A, 439, 129

Cayrel, R. et al., 2004, A\&A, 416, 1117

Chieffi, A. \& Limongi, M., 2004, ApJ, 608, 405

Cowan, J. J. et al., 2002, ApJ, 572, 861

Cowan, J. J. et al., 2005, ApJ, 627, 238

Crawford, J. L., Sneden, C., King, J. R., Boesgaard, A. M. \& Deliyannis, C. P., 1998, AJ, 116, 2489

Freiburghaus, C., Rosswog, S. \& Thielemann, F.-K., 1999, ApJ, 525, L121

Freiburghaus, C. et al., 1999, ApJ, 516, 381

Fröhlich, C. et al., 2006a, ApJ, 637, 415

Fröhlich, C. et al., 2006b, PhRvL, 96, 142502

Fuller, G. M. \& Meyer, B. S., 1995, ApJ, 453, 792

Hill, V. et al., 2002, A\&A, 387, 560

Hoffman, R. D., Woosley, S. E. \& Qian, Y.-Z., 1997, ApJ, 482, 951

Honda, S., Aoki, W., Ishimaru, Y., Wanajo, S. \& Ryan, S. G., 2006, ApJ, 643, 1180

Ishimaru, Y. \& Wanajo, S., 1999, ApJ, 511, L33 
Ivans, I. I., Simmerer, J., Sneden, C., Lawler, J. E., Cowan, J. J., Gallino, R. \& Bisterzo, S., 2006, ApJ, 645, 613

Johnson, J. A. \& Bolte, M., 2002, ApJ, 579, 616

Kitaura, F. S., Janka, H.-T. \& Hillebrandt, W., 2006, A\&A, 450, 345

Mathews, G. J., Bazan, G. \& Cowan, J. J., 1992, ApJ, 391, 719

Mayle, R. \& Wilson, J. R., 1988, ApJ, 334, 909

McLaughlin, G. C. \& Surman, R., 2005, NuPhA, 758, 189

Meyer, B. S. \& Brown, J. S., 1997, ApJS, 112, 199

Meyer, B. S., Mathews, G. J., Howard, W. M., Woosley, S. E. \& Hoffman, R. D., 1992, ApJ, 399, 656

Meynet, G., Ekström, S. \& Maeder, A., 2006, A\&A, 447, 623

Ning, H., Qian, Y.-Z. \& Meyer, B. S., 2007, ApJ, 667, L159

Nomoto, K., 1984, ApJ, 277, 791

Nomoto, K., 1987, ApJ, 322, 206

Otsuki, K., Honda, S., Aoki, W., Kajino, T. \& Mathews, G. J., 2006, ApJ, 641, L117

Pruet, J., Woosley, S. E. \& Hoffman, R. D., 2003, ApJ, 586, 1254

Pruet, J., Woosley, S. E., Buras, R., Janka, H. T. \& Hoffman, R. D., 2005, ApJ, 623, 325

Pruet, J., Hoffman, R. D., Woosley, S. E., Janka, H. T. \& Buras, R., 2006, ApJ, 644, 1028

Qian, Y.-Z., 2000, ApJ, 534, L67

Qian, Y.-Z. \& Wasserburg, G. J., 2002, ApJ, 567, 515

Qian, Y.-Z. \& Wasserburg, G. J., 2003, ApJ, 588, 1099
Qian, Y.-Z. \& Wasserburg, G. J., 2007, PhR, 442, 237

Qian, Y.-Z. \& Woosley, S. E., 1996, ApJ, 471, 331

Qian, Y.-Z., Fuller, G. M., Mathews, G. J., Mayle, R. W., Wilson, J. R. \& Woosley, S. E., 1993, PhRvL, 71, 1965

Ritossa, C., García-Berro, E. \& Iben, Jr, I., 1999, ApJ, 515, 381

Sneden, C. et al., 2003, ApJ, 591, 936

Takahashi, K., Witti, J. \& Janka, H.-T., 1994, A\&A, 286, 857

Thompson, T. A., 2003, ApJ, 585, L33

Thompson, T. A., Burrows, A. \& Meyer, B. S., 2001, ApJ, 562, 887

Tominaga, N., Umeda, H. \& Nomoto, K., 2007, ApJ, 660, 516

Wanajo, S., 2006, ApJ, 647, 1323

Wanajo, S. \& Ishimaru, Y., 2006, NuPhA, 777, 676

Wanajo, S., Tamamura, M., Itoh, N., Nomoto, K., Ishimaru, Y., Beers, T. C. \& Nozawa, S., 2003, ApJ, 593, 968

Westin, J., Sneden, C., Gustafsson, B. \& Cowan, J. J., 2000, ApJ, 530,783

Wheeler, J. C., Cowan, J. J. \& Hillebrandt, W., 1998, ApJ, 493, L101

Witti, J., Janka, H.-T. \& Takahashi, K., 1994, A\&A, 286, 841

Woosley, S. E. \& Baron, E., 1992, ApJ, 391, 228

Woosley, S. E. \& Hoffman, R. D., 1992, ApJ, 395, 202

Woosley, S. E. \& Weaver, T. A., 1995, ApJS, 101, 181

Woosley, S. E., Wilson, J. R., Mathews, G. J., Hoffman, R. D. \& Meyer, B. S., 1994, ApJ, 433, 229 